treatment provided for patients with TB, even though the quality of critical care has improved dramatically since the beginning of this study [2]. In Korea, directly observed therapy (DOT) is not routinely followed [1]. Instead, daily therapy is given, even during the continuation phase, in almost $100 \%$ of patients. Ethambutol is usually prescribed for the entire 6 months. These are probably the reasons why TB has been controlled successfully in Korea, despite the fact that DOT has not been adopted. The prevalence of TB dropped dramatically from $5.1 \%$ in 1965 to $1.0 \%$ in 1995 [3]. In our present study [2], because all patients were supported by mechanical ventilation, anti-TB drugs were taken via nasogastric tube or gastrostomy tube, and it was not possible to skip medications.

As D. Aggarwal commented, the mortality rate was very high in this study. However, drug resistance, especially multidrugresistance, was not an important risk factor for mortality because only 2 out of 90 patients had multidrug-resistant tuberculosis. Even though old age was revealed to be a poor prognostic factor in patients with tuberculosis with respiratory failure who required mechanical ventilation, as D. Aggarwal mentioned, old age is already a well-known poor prognostic factor in several diseases. The most important finding in this study is that corticosteroid use may reduce mortality in patients with severe pulmonary tuberculosis with respiratory failure who require mechanical ventilation. However, because of the inherent limitations of this retrospective study, investigators cannot conclude that steroids are beneficial in reducing mortality in these patients. A prospective randomised study should be conducted to further explore this topic.

\section{Y.J. Kim and T.S. Shim}

Division of Pulmonary and Critical Care Medicine, University of Ulsan College of Medicine, Asan Medical Center, Seoul, South Korea.

\section{STATEMENT OF INTEREST}

None declared.

\section{REFERENCES}

1 Seung KJ, Bai GH, Kim SJ, Lew WJ, Park SK, Kim JY. The treatment of tuberculosis in South Korea. Int J Tuberc Lung Dis 2003; 7: 912-919.

2 Kim YJ, Pack KM, Jeong E, et al. Pulmonary tuberculosis with acute respiratory failure. Eur Respir J 2008; 32: 1624-1629.

3 Hong YP, Kim SJ, Lew WJ, Lee EK, Han YC. The seventh nationwide tuberculosis prevalence survey in Korea, 1995. Int J Tuberc Lung Dis 1998; 2: 27-36.

DOI: $10.1183 / 09031936.00125708$

\title{
High incidence of sputum smear negative tuberculosis during HAART in Burkina Faso
}

\section{To the Editors:}

Tuberculosis (TB) and HIV co-infection is a public health priority in sub-Saharan Africa, where TB is the leading cause of death among HIV infected patients and the first manifestation of the HIV infection. [1-3]. An unprecedented global effort allowed increasing access to antiretroviral treatment in Africa, where $>2$ million persons received highly active antiretroviral therapy (HAART) at the end of 2007 [1].

There is evidence that HAART reduces the risk of TB, in both industrialised [4] and resource-limited countries in subSaharan Africa and south-east Asia [5-9]. However, there is no information on the HAART impact on the incidence of the different TB forms (sputum smear positive pulmonary (SSPPTB), sputum smear negative (SSN-PTB) and extrapulmonary (ЕРTB)).

We have measured TB incidence in a retrospective cohort of HIV infected persons who started HAART in four HIV/AIDS treatment centres in Ouagadougou, Burkina Faso. All consecutive HIV-seropositive patients aged $\geqslant 15 \mathrm{yrs}$, TB-free at HAART initiation, with a follow-up of 12 months or longer were included in the analysis. TB diagnosis was based on internationally accepted criteria [10]. Pulmonary TB (PTB) was diagnosed by microscopy (SSP-PTB) or, in the case of SSN-PTB, on algorithms requiring all the following criteria: 1) chest radiography compatible with active $\mathrm{TB} ; 2$ ) unresponsiveness to $\geqslant 1$ course of large-spectrum antibiotic; and 3) clinician's decision to prescribe a full course of anti-TB therapy. EPTB was based on the clinician's decision to prescribe a full course of anti-TB therapy on the basis of evocative clinical signs, radiological findings and biochemistry of body fluids. Routine culture for Mycobacterium tuberculosis is not available in Burkina Faso.

A cohort of 2,383 HIV-seropositive persons were followed-up for a mean period of $836.1 \pm 443.4$ days. More than a half were classified as World Health Organization (WHO) stage III or IV and $83 \%$ had a CD4 cell count of $<200$ cells $\cdot \mu L^{-1}$ at HAART initiation. A total of 70 TB cases were diagnosed, including 18 (26\%) SSP-PTB, 25 (36\%) SSN-PTB and 27 (38\%) EPTB. Among the 27 EPTB cases, the most frequent TB sites were lymph node $(12(44 \%)$ cases), pleura (5 $(18.5 \%)$ cases) and peritoneum (4 (15\%) cases).

TB incidence declined from 2.80 (95\% confidence interval (CI) $1.60-4.54)$ in the first trimester of HAART to 0.05 (95\% CI $0.01-$ 0.16 ) cases per 100 person $^{-1} \cdot \mathrm{yr}^{-1}$ for $\geqslant 12$ months after its initiation among SSN-PTB patients, from 1.40 (95\% CI 0.60-2.75) to 0.05 (95\% CI $0.01-0.16)$ cases per 100 person $^{-1} \cdot \mathrm{yr}^{-1}$ among SSP-PTB patients, and from 1.57 (95\% CI 0.72-2.99) to 0.07 (95\% CI 0.02 0.19 ) cases per 100 person $^{-1} \cdot \mathrm{yr}^{-1}$ among EPTB patients (fig. 1). 
The present study results demonstrate that there are two times more SSN-PTB than SSP-PTB during early HAART. The decreasing trend was similar for SSP-PTB and SSN-PTB; because the former is a specific indicator for TB incidence, therefore most SSN-PTB patients (being diagnosed largely on radiological ground) were likely to be true TB cases. EPTB incidence also showed a declining trend, an important clue in resource-limited countries considering the high cost and complexity of instruments necessary for the diagnosis and follow-up of such cases.

National TB programmes in resource-limited settings should be aware that most cases arising during the initial HAART stages are PTB cases that are negative at microscopy examination. The adoption of stringent diagnostic criteria would likely result in gross under-diagnosis of TB. High SSN-PTB incidence implies an increased workload, as diagnosis of this condition is significantly more demanding. Although culture has been advocated to increase microbiological sensitivity [3], the fragile health infrastructure in most resource-limited settings seems to make this an unrealistic goal in the midterm. Radiological facilities should as a minimum be strengthened. Finally, research on differential diagnosis of suspect EPTB cases and the identification of appropriate diagnostic procedures for this condition is warranted.

Overall, the high incidence of tuberculosis during early highly active antiretroviral therapy, with most cases presenting with negative microscopy, clearly indicates that HIV programmes in resource-limited settings need to invest more on tuberculosis diagnosis and care to reduce individual morbidity and mortality and to prevent nosocomial transmission of the disease.

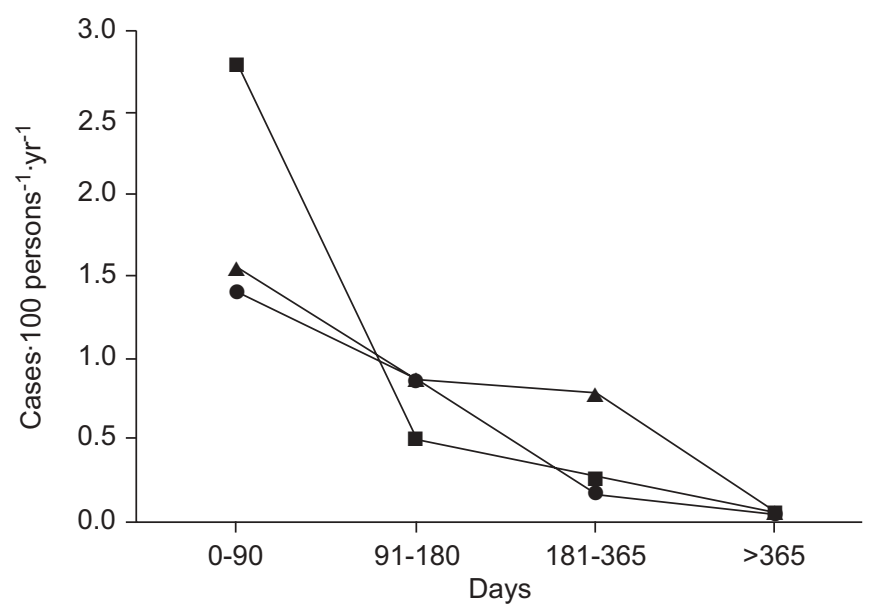

FIGURE 1. Incidence of tuberculosis (TB) among HIV seropositive patients (cases per 100 persons $^{-1} \cdot \mathrm{yrs}^{-1}$ ) by timing after initiation of highly active antiretroviral therapy and site of the disease in Burkina Faso in the period 2005-2007. • : sputum smear positive pulmonary TB (PTB); $\mathbf{\square}$ : sputum smear negative PTB; extrapulmonary TB.
M. Dembelé*, N. Saleri*" ${ }^{*}$, G.B. Migliori ${ }^{\Uparrow}$, H. Ouedraogo ${ }^{+}$, A.C.C. Carvalho $^{\#}$, M. Ouedraogo ${ }^{\S}$, G. Badoum ${ }^{\S}$ and A. Matteelli ${ }^{\#}$

*National Tuberculosis Program, Ministry of Health, ${ }^{+}$National Research Institute, ${ }^{\S}$ Dept of Pulmonary Care, "Yalgado" National Hospital, University of Ouagadougou, Ouagadougou, Burkina Faso. "Institute of Infectious and Tropical Diseases, University of Brescia, Brescia, and ${ }^{\top}$ World Health Organization Collaborating Centre for Tuberculosis and Lung Diseases, Fondazione S. Maugeri, Care and Research Institute, Tradate, Italy.

\section{STATEMENT OF INTEREST}

None declared.

\section{ACKNOWLEDGEMENTS}

The present authors would like to thank D.A. Hien, I. Zabsonre, T.S. Koala, J. Simporé and B. Cauchoix for their contribution to study design and data collection; R. Centis for revising the manuscript; and the Global Fund, Grant BUR 404-T for financial support.

\section{REFERENCES}

1 WHO, UNAIDS and UNICEF. Towards Universal Access: Scaling Up Priority HIV/AIDS Interventions In The Health Sector. Progress Report: Geneva, World Health Organization. 2008

2 World Health Organization. The global plan to stop TB 2006-2015. http:/ / www.stoptb.org/globalplan/plan_main.asp Date last accessed: 18 June 2008.

3 Dye C, Floyd K, Uplekar M. Global Tuberculosis Control: Surveillance, Planning, Financing. WHO Report 2008. Geneva, World Health Organization, 2008. (WHO/HTM/ TB/2008.393).

4 Girardi E, Antonucci G, Vanacore P, et al. Impact of combination antiretroviral therapy on the risk of tuberculosis among persons with HIV infection. AIDS 2000; 14: 1985-1991.

5 Badri M, Wilson D, Wood R. Effect of highly active antiretroviral therapy on incidence on tuberculosis in South Africa: a cohort study. Lancet 2002; 359: 2059-2064.

6 Lawn SD, Myer L, Bekker LG, Wood R. Burden of tuberculosis in an antiretroviral treatment programme in subSaharan Africa: impact on treatment outcomes and implications for tuberculosis control. AIDS 2006; 20: 1605-1612.

7 Moore D, Liechty C, Ekwaru P, et al. Prevalence, incidence and mortality associated with tuberculosis in HIV-infected patients initiating antiretroviral therapy in rural Uganda. AIDS 2007; 21: 713-719.

8 Moh R, Danel C, Messou E, et al. Incidents and determinants of mortality and morbidity following early antiretroviral therapy initiation in HIV infected adults in West Africa. AIDS 2007; 21: 2483-2491.

9 Bonnet MM, Pinoges LL, Varaine FF, et al. Tuberculosis after HAART initiation in HIV-positive patients from five countries with a high tuberculosis burden. AIDS 2006; 20: 1275-1279.

10 Tuberculosis Coalition for Technical Assistance. International Standards for Tuberculosis Care (ISTC). The Hague: Tuberculosis coalition for technical assistance, 2006.

DOI: $10.1183 / 09031936.00106308$ 